\title{
Early magnetic resonance imaging control after temporomandibular joint arthrocentesis
}

David Faustino Ângelo, $\underline{\text { Rita Sousa, }},{ }^{1} \underline{\text { Isabel Pinto }},{ }^{2}$ David Sanz, ${ }^{2}$ F. Monje Gil, ${ }^{3}$ and Francisco Salvado

Department of Stomatology, Santa Maria University Hospital - North Lisbon Hospital, Lisbon, Portugal

${ }_{1}^{1}$ Department of Neuroradiology, Santa Maria University Hospital - North Lisbon Hospital, Lisbon, Portugal

2 Department of Maxilofacial, Coimbra Hospital and University Centre, Coimbra, Portugal

3 Department of Oromaxilofacial, Hospital Infanta Cristina, Badajoz, Spain

Address for correspondence: Dr. David Faustino Ângelo, Department of Stomatology, Santa Maria University Hospital - North Lisbon Hospital, Av. Prof. Egas Moniz, 1649-035 Lisbon. E-mail: david.serrano.angelo.phd@gmail.com

Copyright : @ 2015 Annals of Maxillofacial Surgery

This is an open access article distributed under the terms of the Creative Commons Attribution-NonCommercial-ShareAlike 3.0 License, w hich allow s others to remix, tw eak, and build upon the w ork non-commercially, as long as the author is credited and the new creations are licensed under the identical terms.

\section{Abstract}

Temporomandibular joint (TMJ) lysis and lavage arthrocentesis with viscosupplementation are an effective treatment for acute disc displacement (DD) without reduction. Clinical success seems to be related to multiple factors despite the lack of understanding of its mechanisms. The authors present a case report of 17-year-old women with acute open mouth limitation $(12 \mathrm{~mm})$, right TMJ pain-8/10 visual analog scale, right deviation when opening her mouth. The clinical and magnetic resonance imaging (MRI) diagnosis was acute DD without reduction of right TMJ. Right TMJ arthrocentesis was purposed to the patient with lysis, lavage, and viscosupplementation of the upper joint space. After 5 days, a new MRI was performed to confirm upper joint space distension and disc position. Clinical improvement was obtained 5 days and 1 month after arthrocentesis. Upper joint space increased $6 \mathrm{~mm}$ and the disc remained displaced. We report the first early TMJ MRI image postoperative, with measurable upper joint space.

Keywords: Temporomandibular joint arthrocentesis, temporomandibular joint magnetic resonance imaging postoperative, temporomandibular joint upper joint space distension

\section{INTRODUCTION}

Temporomandibular disorders (TMD) include a wide range of pathological conditions of temporomandibular joint (TMJ) and related musculoskeletal structures.[1] They are frequently misdiagnosed and mistreated in clinical practice, negatively affecting patients' lives. TMD may be divided into two main groups: Intra-articular and extraarticular disorders. The most common intra-articular derangements are disc displacement (DD) with reduction, DD without reduction and degenerative joint disease.[ㄹ, $\underline{3}]$ Noninvasive methods including medication, physiotherapy, and occlusal splints are the first treatment option. When these methods fail surgical approach may be necessary.[$[\underline{4}, \underline{5}, \underline{6}, \underline{7}]$ One of the minimal invasive treatment procedures consists of arthrocentesis lysis and lavage since it is effective in reducing intra-articular adhesions and releasing the articular disc, reducing pain and restoring normal mandibular function. It also plays a role by reducing inflammatory cytokines in synovial fluid. $[\underline{8}, \underline{9}, \underline{10}]$ In addition, TMJ arthrocentesis also reduces intracapsular pressure by a hydraulic distension of the upper joint space. $[11,12]$ Hyaluronic acid is the main component of the synovial fluid and has a great importance in joint lubrication, reducing friction within the intra-articular space with a "bearing effect" against impact, and its analgesic effect. $[\underline{13}, \underline{14}]$ High TMJ load can compromise capillary perfusion.[15, $\underline{16}]$ Temporary hypoxia conduces to free radicals' release and is responsible for hyaluronic acid degradation, decreasing synovial viscosity, reducing intraarticular lubrification, contributing to a pathologic condition by damaging extracellular and intracellular molecules. $[\underline{15}, \underline{17}, \underline{18}]$ Although there is no solid evidence of the benefits of the use of intra-articular acid hyaluronic injection, many authors suggest clinical long-term improvement of TMJ symptoms after its use. $[\underline{[}, \underline{9}, \underline{10}, \underline{19}, \underline{20}, \underline{21}, \underline{22}]$ 
Magnetic resonance imaging (MRI) is the gold standard exam to diagnose intra-articular changes.[23] TMJ disc plays an important role in TMJ function. The evaluation of its position and shape is crucial for treatment decision. $[\underline{8}, \underline{11}, \underline{23}]$ Moses et al. investigated TMJ disc position with MRI before and after arthroscopic lysis and lavage. They report that in spite of only $8 \%$ of the luxated discs achieved reduction, $92 \%$ of the patients had a significant decrease in pain and restoration of normal mandibular function.[24] Therefore, TMJ arthrocentesis clinical success seems to be related to multiple factors despite the lack of understanding of its mechanisms.

\section{CASE REPORT}

A 17-year-old woman attended to our consultation after sudden onset $(4 \mathrm{~h})$ of right TMJ pain and restricted mouth opening, not relieved with ibuprofen. The patient referred a prior history of asymptomatic right TMJ clicking for the past 2 years and orthodontic treatment 3 years before. She denied any trauma or infection episode. Clinical examination revealed 8/10 visual analogue scale right TMJ pain and no pain in left TMJ. Maximal interincisal mouth opening was $12 \mathrm{~mm}$ with right deviation [Figure 1a]. Lateral and forward excursions were limited due to pain. Right retrodiscal tissue palpation was painful and no clicks were present. We found no muscular aches. Clinical diagnosis was an acute DD without reduction of right TMJ, confirmed by MRI (axial T1, coronal T2, sagittal DP and T2 spectral adiabatic inversion recovery-close and open mouth).

\section{Management and outcome}

We performed right TMJ arthrocentesis after local anesthesia with lidocaine blocking the auriculotemporal nerve. As performed in the classical technique, a tragocantal line was drawn, and the first needle was inserted $10 \mathrm{~mm}$ anterior and $2 \mathrm{~mm}$ inferior of the tragus. $1.8 \mathrm{~mL}$ of lidocaine with epinephrine 1:80.000 was injected to widen the upper joint space. The second needle was inserted $20 \mathrm{~mm}$ anterior and $7 \mathrm{~mm}$ inferior of the tragus. Then the joint was washed with $250 \mathrm{cc}$ of Ringer Lactate. At the end, $1.5 \mathrm{cc}$ of high stability hyaluronic acid (Durolane SJ) was injected. Five days after arthrocentesis the patient was asymptomatic, with maximal interincisal mouth opening of $32 \mathrm{~mm}$, without deviation [igure 1b]. No clicks were present. An early postoperative MRI showed an increased upper joint space of $6 \mathrm{~mm}$ [Figure 2] with a consequent more pronounced anteroinferior displacement of the condyle for the same degree of mouth opening. The disc remained anteriorly displaced but with reduction [ Figure 3].

\section{DISCUSSION}

As previously described in the literature, TMJ arthrocentesis is an effective treatment for acute DD without reduction, reducing pain and limited mouth opening. $[\underline{8}, \underline{19}, \underline{25}, \underline{26}]$ However, disc repositioning does not seem to play a major role since many authors report clinical success without changing disc position.[24] Many theories have been suggested for clinical improvement after TMJ arthrocentesis unrelated to disc position. One of the theories concerns the intra-articular fluid flow rate and intracapsular pressure distribution during arthrocentesis. Variable fluid dynamic characteristics contribute for different therapeutic results: Increased flow is associated with more effective lavage with increased pain reduction.[27] Few studies demonstrated that the irrigation pressure is effective in reducing adhesions. However, in clinical practice, it is not easy to control flow rate or measure intracapsular pressure. Alternatively, pain reduction might be due to the decrease of inflammatory cytokines, namely bradykinin and interleukin-6.[묘, $\underline{26}$. Once again, the measurement of synovial fluid cytokines in clinical practice is not feasible. Nitzan proposed that the removal of vacuum intra-articular effect and the change of synovial fluid viscosity could explain arthocentesis success while improving disc and condyle mobility.[11, 17$]$ According to Nitzan theory of hyaluronic acid,[11] we hypothise that the clinical improvement in this patient is explained by an improved capillary perfusion due to reduced intra-articular pressure, improving intra-articular nutrient perfusion and consequent joint remodeling and adaptation. MRI measurement of the upper joint space before and after arthrocentesis could be a reliable and reproducible method for monitoring and evaluation of the clinical success of the technique. Therefore the measurement of upper joint space after lavage and hyaluronic acid upper joint injection could predict clinical benefits.

\section{CONCLUSIONS}

Hydraulic distension could be an important measure to predict clinical success. Hydraulic distension measurement could explain more about TMJ arthrocentesis mechanisms. Long-term studies would be required to assess the relevance of upper joint distension, but it seems that hydraulic distension plays an important role in clinical success. 
Financial support and sponsorship

Nil.

\section{Conflicts of interest}

There are no conflicts of interest.

\section{REFERENCES}

1. Tvrdy P, Heinz P, Pink R. Arthrocentesis of the temporomandibular joint: Biomedical papers of the Medical Faculty of the University Palacky, Olomouc, Czechoslovakia. 2013;159:31-34.

2. Dworkin SF, LeResche L. Research diagnostic criteria for temporomandibular disorders: Review, criteria, examinations and specifications, critique. J Craniomandib Disord. 1992;6:301-55. [PubMed: 1298767]

3. Manfredini D, Guarda-Nardini L, Winocur E, Piccotti F, Ahlberg J, Lobbezoo F. Research diagnostic criteria for temporomandibular disorders: A systematic review of axis I epidemiologic findings. Oral Surg Oral Med Oral Pathol Oral Radiol Endod. 2011;112:453-62. [PubMed: 21835653]

4. Al-Belasy FA, Dolwick MF. Arthrocentesis for the treatment of temporomandibular joint closed lock: A review article. Int J Oral Maxillofac Surg. 2007;36:773-82. [PubMed: 17582743]

5. Stein JI. TMJ arthrocentesis. A conservative surgical alternative. N Y State Dent J. 1995;61:68-76.

[PubMed: 8524521]

6. Tozoglu S, Al-Belasy FA, Dolwick MF. A review of techniques of lysis and lavage of the TMJ. Br J Oral Maxillofac Surg. 2011;49:302-9. [PubMed: 20471143]

7. Sahlström LE, Ekberg EC, List T, Petersson A, Eriksson L. Lavage treatment of painful jaw movements at disc displacement without reduction. A randomized controlled trial in a short-term perspective. Int J Oral Maxillofac Surg. 2013;42:356-63. [PubMed: 23140985]

8. Monje-Gil F, Nitzan D, González-Garcia R. Temporomandibular joint arthrocentesis. Review of the literature. Med Oral Patol Oral Cir Bucal. 2012;17:e575-81. [PMCID: PMC3476018] [PubMed: 22322493]

9. Nitzan DW. Arthrocentesis - Incentives for using this minimally invasive approach for temporomandibular disorders. Oral Maxillofac Surg Clin North Am. 2006;18:311-28, vi. [PubMed: 18088835]

10. Nitzan DW, Price A. The use of arthrocentesis for the treatment of osteoarthritic temporomandibular joints. J Oral Maxillofac Surg. 2001;59:1154-9. [PubMed: 11573170]

11. Nitzan DW, Dolwick MF. An alternative explanation for the genesis of closed-lock symptoms in the internal derangement process. J Oral Maxillofac Surg. 1991;49:810-5. [PubMed: 2072192]

12. Nitzan DW, Etsion I. Adhesive force: The underlying cause of the disc anchorage to the fossa and/or eminence in the temporomandibular joint - A new concept. Int J Oral Maxillofac Surg. 2002;31:94-9. [PubMed: 11936408]

13. De Riu G, Stimolo M, Meloni SM, Soma D, Pisano M, Sembronio S, et al. Arthrocentesis and temporomandibular joint disorders: Clinical and radiological results of a prospective study. Int J Dent. 2013;2013:790648. [PMCID: PMC3844254] [PubMed: 24319462]

14. Bonotto D, Machado E, Cunali RS, Cunali PA. Viscosupplementation as a treatment of internal derangements of the temporomandibular joint: Retrospective study. Rev. dor. 2014;15:2-5.

15. Kawai Y, Lee MC, Kubota E. Oxidative stress and temporomandibular joint disorders. Jpn Dent Sci Rev. $2008 ; 44: 145-50$.

16. Nitzan DW. Intraarticular pressure in the functioning human temporomandibular joint and its alteration by uniform elevation of the occlusal plane. J Oral Maxillofac Surg. 1994;52:671-9. [PubMed: 8006730]

17. Nitzan DW, Goldfarb A, Gati I, Kohen R. Changes in the reducing power of synovial fluid from temporomandibular joints with "anchored disc phenomenon" J Oral Maxillofac Surg. 2002;60:735-40. 
[PubMed: 12089684]

18. Milam SB, Zardeneta G, Schmitz JP. Oxidative stress and degenerative temporomandibular joint disease: A proposed hypothesis. J Oral Maxillofac Surg. 1998;56:214-23. [PubMed: 9461148]

19. Emshoff R, Rudisch A, Bösch R, Strobl H. Prognostic indicators of the outcome of arthrocentesis: A shortterm follow-up study. Oral Surg Oral Med Oral Pathol Oral Radiol Endod. 2003;96:12-8. [PubMed: 12847438]

20. Ahmed N, Sidebottom A, O’Connor M, Kerr HL. Prospective outcome assessment of the therapeutic benefits of arthroscopy and arthrocentesis of the temporomandibular joint. Br J Oral Maxillofac Surg. 2012;50:745-8. [PubMed: 22381517]

21. Emshoff R, Rudisch A. Determining predictor variables for treatment outcomes of arthrocentesis and hydraulic distention of the temporomandibular joint. J Oral Maxillofac Surg. 2004;62:816-23. [PubMed: 15218559]

22. Sidebottom A, Ahmed N. The role of arthroscopy and arthrocentesis in TMJ management. Face Mouth Jaw Surg. 2012;2:22-8.

23. Barmeir E, Teich S, Gutmcher Z. MRI of the temporomandibular joint - The gold standard. Refuat Hapeh Vehashinayim (1993) 2014;31:19-27. 86. [PubMed:25252468]

24. Moses JJ, Sartoris D, Glass R, Tanaka T, Poker I. The effect of arthroscopic surgical lysis and lavage of the superior joint space on TMJ disc position and mobility. J Oral Maxillofac Surg. 1989;47:674-8.

[PubMed: 2732826]

25. Manfredini D, Rancitelli D, Ferronato G, Guarda-Nardini L. Arthrocentesis with or without additional drugs in temporomandibular joint inflammatory-degenerative disease: Comparison of six treatment protocols*. J Oral Rehabil. 2012;39:245-51. [PubMed: 21999138]

26. Nogami S, Yamauchi K, Kataoka Y, Takano H, Yamashita Y, Takahashi T. Clinical comparison between arthrocentesis and conventional conservative treatment with maxillomandibular fixation for unilateral high condylar fractures. J Oral Rehabil. 2013;41:141-147. [PubMed: 24372314]

27. Xu Y, Lin H, Zhu P, Zhou W, Han Y, Zheng Y, et al. A comparative study between use of arthroscopic lavage and arthrocentesis of temporomandibular joint based on computational fluid dynamics analysis. PLoS One. 2013;8:e78953. [PMCID: PMC3815200] [PubMed: 24223868]

\section{Figures and Tables}

\section{Figure 1}

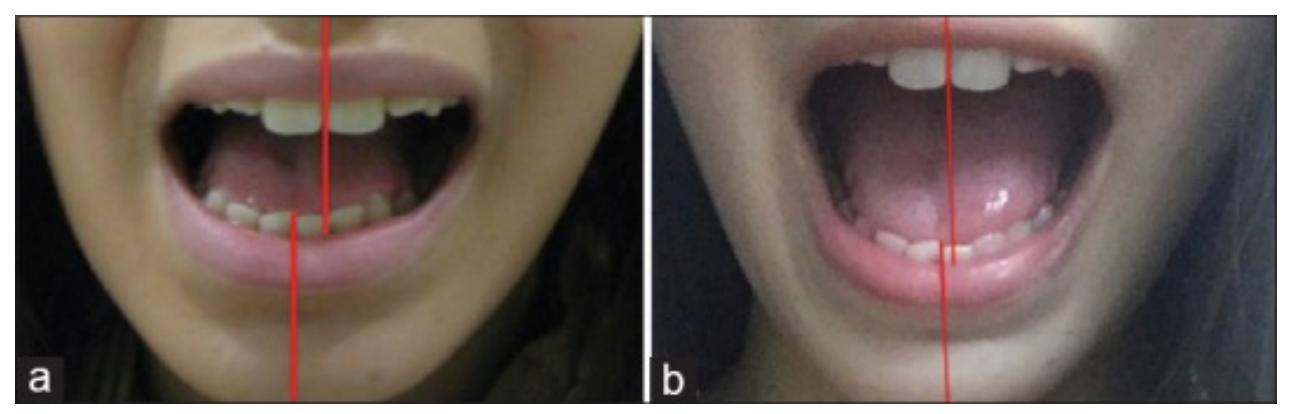

(a) Patient with intense right temporomandibular joint pain and mouth opening limitation to $12 \mathrm{~mm}$ with right deviation.

(b) Patient after temporomandibular joint arthrocentesis and viscosupplementation with $36 \mathrm{~mm}$ mouth opening and partial correction of mouth deviation

Figure 2 


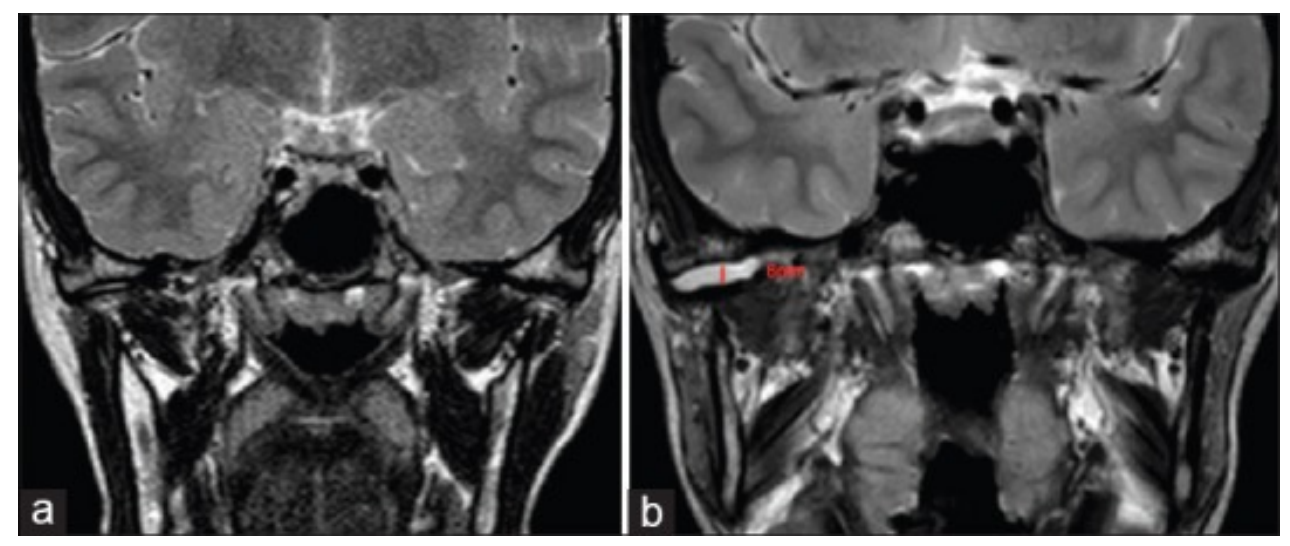

(a) Coronal T2 magnetic resonance imaging (close mouth) before temporomandibular joint arthrocentesis. (b) Coronal T2 (close mouth) 5 days after procedure - upper joint space increased $6 \mathrm{~mm}$

\section{Figure 3}
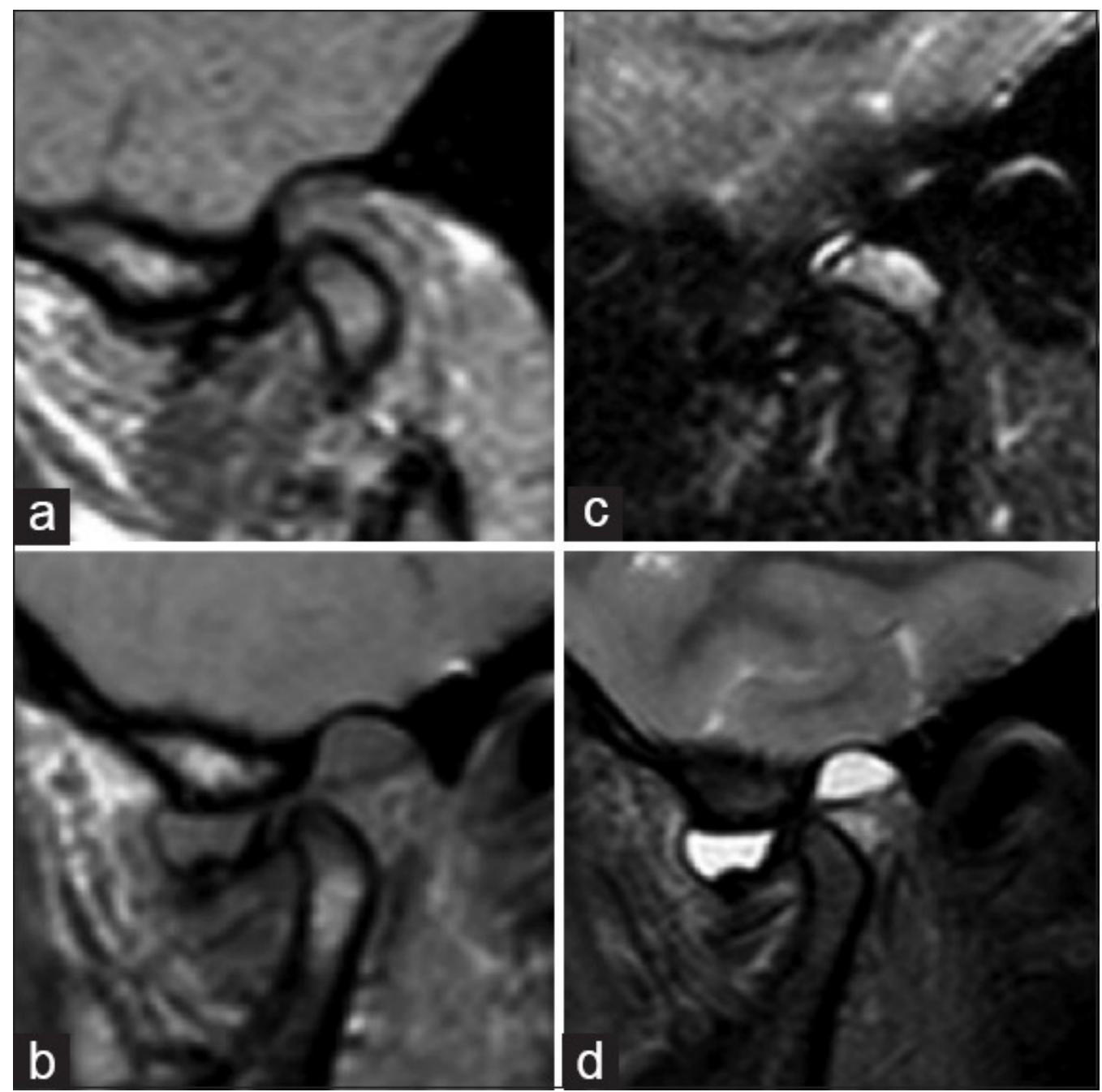

Preoperative MRI (1, 5 Tesla - $\mathrm{a}$ and $\mathrm{b})$ and postoperative risk of malignancy index (3 Tesla - c and d) sagittal PD (a and c) and T2 spectral adiabatic inversion recovery (b and d) sagittal open mouth magnetic resonance images $(9 \mathrm{~mm})-$ greater amplitude of condyle motion after procedure, increased upper articular compartment, persistent anterior disc displacement without reduction

Articles from Annals of Maxillofacial Surgery are provided here courtesy of Wolters Kluwer -- Medknow Publications 